https://helda.helsinki.fi

\title{
Anti-inflammatory medication after cataract surgery and posterior capsular opacification
}

\section{Hecht, Idan}

2020-07

Hecht , I , Karesvuo , P , Achiron , A , Elbaz , U , Laine , I \& Tuuminen , R 2020 , '

Anti-inflammatory medication after cataract surgery and posterior capsular opacification ' ,

American Journal of Ophthalmology , vol. 215 , pp. 104-111 . https://doi.org/10.1016/j.ajo.2020.02.007

http://hdl.handle.net/10138/326422

https://doi.org/10.1016/j.ajo.2020.02.007

cc_by_nc_nd

acceptedVersion

Downloaded from Helda, University of Helsinki institutional repository.

This is an electronic reprint of the original article.

This reprint may differ from the original in pagination and typographic detail.

Please cite the original version. 


\section{Journal Pre-proof}

Anti-inflammatory medication after cataract surgery and posterior capsular opacification

Idan Hecht, Petteri Karesvuo, Asaf Achiron, Uri Elbaz, Ilkka Laine, Raimo Tuuminen

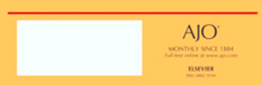

PII:

S0002-9394(20)30060-X

DOI:

https://doi.org/10.1016/j.ajo.2020.02.007

Reference: AJOPHT 11230

To appear in: American Journal of Ophthalmology

Received Date: 15 November 2019

Revised Date: 1 February 2020

Accepted Date: 5 February 2020

Please cite this article as: Hecht I, Karesvuo P, Achiron A, Elbaz U, Laine I, Tuuminen R, Antiinflammatory medication after cataract surgery and posterior capsular opacification, American Journal of Ophthalmology (2020), doi: https://doi.org/10.1016/j.ajo.2020.02.007.

This is a PDF file of an article that has undergone enhancements after acceptance, such as the addition of a cover page and metadata, and formatting for readability, but it is not yet the definitive version of record. This version will undergo additional copyediting, typesetting and review before it is published in its final form, but we are providing this version to give early visibility of the article. Please note that, during the production process, errors may be discovered which could affect the content, and all legal disclaimers that apply to the journal pertain.

(c) 2020 Elsevier Inc. All rights reserved. 


\section{Abstract}

Purpose: To assess the role of anti-inflammatory medication following cataract surgery on the formation of posterior capsular opacification.

\section{Design: Cohort study.}

Methods: A retrospective registry analysis of 25,818 consecutive cases who underwent cataract surgery between the years 2014 and 2018 at Helsinki University Hospital in Finland. $\mathrm{Nd}$ :YAG laser capsulotomy rates were compared between patients treated postoperatively with topical steroids, non-steroidal anti-inflammatory medications (NSAIDs), or their combination. Kaplan-Meier and Cox regression analyses were used. A single eye of each patient was included. Main outcomes were confirmed against a second independent dataset.

Results: 13,368 patients were included in the analysis with a mean age of $73.2 \pm 9.7$ years and $61.7 \%$ were female. Pseudoexfoliation was noted in $10.1 \%$ of cases. The mean follow-up time was $22.8 \pm 15.7$ months. Patients were treated with steroid monotherapy ( $28.9 \%$ of cases), NSAIDs monotherapy (62.2\%), or a combination of both (8.9\%). Treatment with steroids resulted in significantly lower Nd:YAG capsulotomy rates compared to NSAIDs (HR 0.76, 95\% CI 0.62-0.93, $P=0.009$ ). Treatment with combination therapy of steroids and NSAIDs showed no added benefit over steroid monotherapy (HR 1.11, 95\% CI 0.68-1.80, $P=0.674)$. Cox regression analysis adjusted for patients' age, gender, pseudoexfoliation, and risk stratification remained significantly predictive for lower capsulotomy rates with steroid treatment over NSAIDs (HR 0.70, 95\% CI 0.52-0.88, $P=0.001$ ).

Conclusions: Postoperative treatment with steroids among patients undergoing uncomplicated cataract surgery was associated with lower rates of clinically significant posterior capsule opacification compared to treatment with NSAIDs alone. Combination therapy of steroids and NSAIDs had no added benefit over steroids alone.

Key words: anti-inflammatory medication; cataract surgery; NSAIDs; PCO; steroids 


\section{Anti-inflammatory medication after cataract surgery and posterior capsular opacification}

Idan $\mathrm{Hecht}^{1,2}$; Petteri Karesvuo ${ }^{1,3}$; Asaf Achiron ${ }^{4}$; Uri Elbaz ${ }^{5}$; Ilkka Laine ${ }^{1,6}$; Raimo Tuuminen ${ }^{1,7}$

${ }^{1}$ Helsinki Retina Research Group, University of Helsinki, Helsinki, Finland

${ }^{2}$ Department of Ophthalmology, Shamir Medical Center and Sackler School of Medicine, Tel Aviv

University, Tel Aviv, Israel

${ }^{3}$ Department of Ophthalmology, Helsinki University Hospital, Helsinki, Finland

${ }^{4}$ Department of Ophthalmology, Wolfson Medical Center, Holon and Sackler School of Medicine,

Tel Aviv University, Tel Aviv, Israel

${ }^{5}$ Department of Ophthalmology, Rabin Medical Center, Petach-Tikva, and Sackler School of Medicine, Tel Aviv University, Tel Aviv, Israel

${ }^{6}$ Department of Electrical Engineering and Automation, Aalto University, Helsinki, Finland

${ }^{7}$ Eye Centre, Kymenlaakso Central Hospital, Kotka, Finland

Short Title: Anti-inflammatory medication and PCO

Word count: 3044 (Abstract: 249)

Financial Support: None.

Conflict of Interest: no conflicting relationship exists for any author.

Corresponding author: Idan Hecht, MD. Department of Ophthalmology, Shamir Medical Center.

Be'er Ya'akov, 70300, Israel. ORCID ID: 0000-0001-5634-0432. Phone: +972-8-9779620 Fax:

+972-8-9779627 Email: Idanhe@gmail.com 


\section{Introduction}

Cataract surgery is one of the most common surgeries in the world and the most common procedure performed in the United States. ${ }^{1}$ Topical anti-inflammatory drops (steroids and non-steroidal antiinflammatory drugs [NSAIDs]) are typically used for one to three months following the operation. They suppress the inflammatory reaction and reduce postoperative pain, discomfort and complications such as pseudophakic cystoid macular edema. ${ }^{2}$

Posterior capsular opacification (PCO) remains among the most common complications after cataract surgery. ${ }^{3-5}$ It may cause reduced vision and it is estimated to occur in approximately 5-10\% of cases. ${ }^{6,7}$ Although PCO can be easily treated with neodymium-doped yttrium aluminum garnet (Nd:YAG) laser capsulotomy, the procedure is associated with additional costs and possible complications such as a transient increase in intraocular pressure, lens pitting, lens subluxation and retinal detachment. ${ }^{8,9}$

Most studies aiming at reducing PCO formation have focused on various changes to the shape and material of the intraocular lens (IOL). ${ }^{10-12}$ However, in vitro, ex vivo and animal models have suggested that inflammatory mediators play a major role in regulating lens epithelial cell (LEC) proliferation associated with PCO formation. ${ }^{13,14}$ As topical steroids and NSAIDs are common antiinflammatory treatments following cataract removal, we aimed to assess Nd:YAG laser capsulotomy rates after uncomplicated cataract surgery treated postoperatively by different antiinflammatory medications. 


\section{Material and Methods}

This study was a registry-based retrospective cohort study of consecutive cataract surgeries performed at the Helsinki University Hospital, Helsinki, Finland. The study was approved by the Helsinki and Uusimaa Hospital District (Research permission §42/2019; HUS/466/2019) and was in adherence with the tenets of the Declaration of Helsinki.

\section{Data acquisition}

The study included patients who underwent uncomplicated cataract extraction from 1.1.2014 to 31.12.2018 at the Helsinki University Hospital. Data was collected from the operating room management system (BCB Medical, cataract database, Turku, Finland).

Age, gender, laterality, IOL type and diopter power (D), preoperative risk assessment (according to the referral to surgery, graded from 1 to 3), ocular comorbidities (pseudoexfoliation, glaucoma [according to the glaucoma medication in the medical records]) and poor mydriasis were documented (Table 1). Intraoperative details including use of iris hooks, pupil expansion devises and/or capsular tension ring, complications during the surgery and IOL position (anterior chamber, sulcus, or capsular bag) were also documented and used for exclusion purposes (Table 1). Diabetes (type I or II) and the presence of ocular manifestations such as diabetic maculopathy and retinopathy (mild to moderate background retinopathy, severe [pre-proliferative], or proliferative [PDR] retinopathy) were documented if the patients had any mention of the following ICD-codes [E10.0-E10.9, E11.0-E11.9, H36.00, H36.01, H36.02, H36.03]. Among the included patients, $\mathrm{N}=111$ were treated for proliferative diabetic retinopathy (PDR) or diabetic macular edema (DME) during their visits between 2014 and 2018 in the Department of Ophthalmology, Helsinki University Hospital.

For inclusion purposes, patients were required to have documentation on type of postoperative medical treatment received, and the status of Nd:YAG capsulotomy during the follow-up period. Exclusion criteria were iris prolapse, anterior / posterior capsule rupture at any stage of the surgery, intraocular position other than the capsular bag, IOL power outside 6-30 diopters, or patient age under 18 years at the time of the operation. Only cases in which the Tecnis ${ }^{\circledR}$ (Johnson \& Johnson Surgical Vision Inc., Santa Ana, CA) single $\square$ piece acrylic monofocal IOL was used were included, in order to remove IOL type as a possible confounder. Excluded were $3.9 \%$ of cases in which other 
IOLs were used (2.7\% were monofocal single-piece AcrySof IQ ${ }^{\circledR}$ (including toric SN6AT IOLs), and $1.2 \%$ were other IOL types). Only one eye per patient was included in the data analysis. A flow chart of the patient inclusion process is presented in Figure 1.

\section{Surgical technique}

The surgical technique was the standard minimally invasive phacoemulsification technique (Infinity ${ }^{\circledR} /$ Centurion ${ }^{\circledR}$ Vision System, Alcon Laboratories, Inc., Fort Worth, TX) with a 2.4-2.75mm clear corneal incision. A single $\square$ piece acrylic monofocal IOL (Tecnis ${ }^{\circledR}$, Johnson \& Johnson Surgical Vision Inc., Santa Ana, CA) was implanted into the capsular bag. Surgeries were performed by 45 experienced specialist ophthalmologists ( 20 female and 25 male).

\section{Postoperative Care}

The choice of anti-inflammatory medication was at the discretion of the treating physician. Treatment was either with steroids (Pred Forte ${ }^{\circledR}$, prednisolone acetate 10mg/ml [96.1\% of steroids], Allergan, Inc. Irvine, CA; or Oftan Dexa ${ }^{\circledR}$, dexamethasone $1 \mathrm{mg} / \mathrm{ml}$ [3.9\% of steroids], Santen Oy, Tampere, Finland), NSAIDs (Nevanac ${ }^{\circledR}$, nepafenac $1 \mathrm{mg} / \mathrm{ml}[0.4 \%$ of NSAIDs] or $3 \mathrm{mg} / \mathrm{ml}[99.6 \%$ of NSAIDs], Novartis, Basel, Switzerland), or the combination of both. The length of the antiinflammatory treatment was three weeks in $96.2 \%$ of the surgeries; in the rest, the treatment was longer. No subconjunctival or intracameral anti-inflammatory adjunct treatment was used. Indications for $\mathrm{Nd}$ :YAG capsulotomy were decreased corrected distance visual acuity and decreased visual function caused by PCO.

\section{Statistical analysis}

In order to avoid biases arising from between-eye correlation, a single eye of each patient was included $^{15}$; the eye that was operated on first. Unless otherwise specified, data are presented as mean \pm standard deviation (SD). Statistical analysis was performed using IBM SPSS Statistics 25 (SPSS Inc., Chicago, IL). For categorical variables the $\chi^{2}$ test was used. Clinical parameter distributions were tested for normality by the Shapiro-Wilk test. Independent T-test was conducted for continuous variables with a normal distribution and the Mann-Whitney U-test for variables with a non-normal distribution. Kaplan-Meier curves were generated, and Cox regression was used to estimate hazard ratios (HR) for Nd:YAG capsulotomy for different treatments. Adjusted HR's were generated controlling for several possible confounders, using the best subset method, including: age, gender, existence of pseudoexfoliation, and risk stratification. $P$-values less than 0.05 on a twosided test were considered statistically significant. 


\section{Second Independent Dataset}

We repeated the analysis using a second independent dataset of 12,555 cataract surgeries as a method for confirming the main outcomes. For this purpose a registry of cataract surgeries and Nd:YAG laser capsulotomies performed at a different tertiary hospital, Kymenlaakso Central Hospital, Ophthalmology Unit, Kotka, Finland, between 1.1.2010 and 31.10.2018 (Supplemental Table 1). The anti-inflammatory treatment protocol was revised in the Unit in Autumn 2016 from prescribing steroid monotherapy (dexamethasone $1 \mathrm{mg} / \mathrm{ml}$, t.i.d. for a 3-week period following surgery, Oftan Dexa ${ }^{\circledR}$, Santen Oy, Tampere, Finland) to prescribing NSAID monotherapy (diclofenac $1 \mathrm{mg} / \mathrm{ml}$, t.i.d. for a 3-week period following surgery, Dicloabak ${ }^{\circledR}$, Laboratoires Théa, Clermont-Ferrand, France) as the primary anti-inflammatory medication. Inclusion and exclusion criteria as well as the statistical methods were replicated. The study was approved by the Research Director and Chief Medical Officer of the Kymenlaakso Central Hospital and the tenets of the Declaration of Helsinki were followed. 


\section{Results}

\section{Baseline variables}

Included were 13,368 eyes of the same number of patients who underwent uncomplicated cataract surgery. The mean age of patients was $73.2 \pm 9.7$ years and $61.7 \%$ were female. The right eye was operated on in $57.7 \%$ of cases. Mean follow-up time was $22.8 \pm 15.7$ months. Pseudoexfoliation was noted in $10.1 \%$ of cases, poor mydriasis in $13.9 \%$, a pupil expansion device was used in $7.1 \%$ of cases and a high preoperative risk assessment (according to the referral to surgery) was noted in $8.7 \%$ of cases.

Per the inclusion criteria, in all cases a monofocal one-piece Tecnis ${ }^{\circledR}$ IOL was used $(6.7 \%$ were preloaded PCB00 in iTec ${ }^{\circledR}$ delivery system). Patients were treated postoperatively with steroids alone in $28.9 \%$ of cases, NSAIDs alone in $62.2 \%$ of cases, or with a combination of both in $8.9 \%$ of cases.

\section{Nd:YAG capsulotomy rates between steroids, NSAIDs, and their combination}

Overall, 3.5\% of eyes required Nd:YAG capsulotomy during the follow-up period, $0.9 \%$ at 1-year, $2.5 \%$ at 2-years and $6.1 \%$ at 3-years. Treatment with steroids alone compared to NSAIDs alone resulted in significantly lower hazard ratios (HR) for Nd:YAG capsulotomy (HR 0.76, 95\% CI $0.62-0.93, P=0.009,2.3 \%$ vs $2.6 \%$ at two years, $5.0 \%$ vs $7.0 \%$ at three years, Figure 2 ). Treatment with combination therapy of steroids and NSAIDs compared to steroids alone showed no added benefit (HR 1.11, 95\% CI 0.68-1.80, $P=0.674$, Figure 3).

In Cox regression analysis, steroid monotherapy remained predictive for lower capsulotomy rates when compared to NSAID monotherapy, even after adjustment for age, gender, existence of pseudoexfoliation, and risk stratification (HR 0.70, 95\% CI 0.52-0.88, Figure 4, $P=0.001$ ). Cox regression analysis adjusted for similar covariates revealed no added benefit of combination treatment with steroids and NSAIDs over steroid monotherapy (HR 0.93, 95\% CI 0.51-1.35, $P=0.354)$.

\section{Secondary analyses}

Patient age was inversely associated with Nd:YAG capsulotomy rates as the HR for Nd:YAG among patients older than 65 years was 0.58 (95\% CI 0.47-0.71, $P<0.001)$ compared to those $\leq 65$ years. Female gender was significantly associated with higher capsulotomy rates (HR 1.26, 95\% CI 
1.04-1.54, $P=0.018$ ) as illustrated in Supplemental Figure 1. Surgical risk stratification (according to referral to surgery), poor mydriasis, use of pupil expansion device, existence of diabetic macular edema or proliferative diabetic retinopathy were all not significantly associated with Nd:YAG capsulotomy rates (all 95\% CI crossing 1.0, P>0.05). Similarly, neither existence of pseudoexfoliation nor the type of postoperative topical steroid (dexamethasone vs. prednisolone acetate) had any significant association with $\mathrm{Nd}$ :YAG capsulotomy rates (all $P>0.05$ ).

Although cases with documented iris prolapse were excluded, an independent analysis of this factor's association with PCO formation was carried out. In the entire dataset, iris prolapse was documented in 83 cases, among which only one (1.2\%) was documented as requiring posterior capsulotomy postoperatively. This difference was non-significant compared to the rate of posterior capsulotomy among patients without iris prolapse $(3.5 \%, \mathrm{p}=0.264)$.

\section{Second independent dataset}

Main outcomes were confirmed against a second dataset, the registry of cataract surgeries and Nd:YAG laser capsulotomies performed at Kymenlaakso Central Hospital, Kotka, Finland. Included were 12,555 cases, mean age was $75.4 \pm 8.6$ and $63 \%$ were female (supplemental Table 1).

Survival analysis revealed similar results. Eyes primarily treated with steroids (operated on between 1.1.2010 - 15.9.2016) had significantly lower rates of Nd:YAG capsulotomies compared to eyes primarily treated with NSAIDs (operated on between 16.9.2016 - 31.10.2018) (HR 0.43, 95\% CI 0.32-0.57, $P<0.001)$. In a Cox regression model, when patient age and gender were included as confounders, HR for Nd:YAG capsulotomy for eyes primarily treated with steroids was again lower at 0.53 with $95 \% \mathrm{CI}$ of $0.40-0.70(P<0.001)$ compared to eyes primarily treated with NSAIDs. this is illustrated in Supplemental Figure 2. 


\section{Discussion}

In this retrospective registry-based study, we assessed the rates of $\mathrm{Nd}$ :YAG capsulotomies required among patients undergoing uncomplicated cataract extraction treated by different postoperative medical regimens. The results show that patients treated with steroids required significantly less $\mathrm{Nd}$ :YAG capsulotomies than those treated with NSAIDs. In addition, combination therapy of steroids and NSAIDs showed no significant advantage over steroids alone. Given the consistency of the results after accounting for confounders in multivariant analysis, as well as the replicated results in an independent large-scale dataset, and the considerable population size, length of follow-up, and detailed registry used, we believe these conclusions to by justified in this case. Nevertheless, these results should stimulate a large-scale prospective, rigidly controlled trial to properly address the question at hand.

PCO is the most frequent complication of cataract surgery. In recent years several changes made to IOL design and material have been able to reduce PCO formation to some degree, yet it remains a significant problem. ${ }^{16-18}$ Several anti-proliferative and anti-inflammatory medications have been explored in the past, demonstrating the significant role of inflammation behind the development of PCO. ${ }^{17,19}$ A recent animal model study published by Jiang et al. showed that inflammatory cytokine production is involved in the epithelial-mesenchymal transition of residual lens epithelial cells (LECs) in the capsular bag and formation of profibrotic myofibroblasts, eventually leading to PCO. ${ }^{20}$ Symonds et al. used a rat lens model and showed that dexamethasone, but not diclofenac, resulted in the retention of a monolayer of migratory cells and enhanced cell coverage. ${ }^{21}$ Mansfield et al. used a rat PCO model and showed that introduction of dexamethasone caused rapid formation of long, needle-like cells, less extracellular matrix and a monolayerity of cells. ${ }^{22}$ On the other hand, clinical studies on this topic are inconclusive. A two-year prospective randomized control trial in Sweden $(n=180)$ showed similar PCO rates in patients using dexamethasone, diclofenac, or a placebo for three weeks after surgery. ${ }^{23}$ In another clinical study, Inan et al. reported the effect of intracameral diclofenac injection during the hydrodissection stage compared to placebo in a small group of 11 patients, with no significant difference in PCO rates between the groups. ${ }^{24}$

Previous studies analyzing the role of NSAIDs in the formation of PCO are rather experimental or include small sample size that may lack sufficient power. ${ }^{20-24}$ Furthermore, the results of these piloting studies are contradictory. ${ }^{20-24}$ This highlights the importance of real-life evidence with proper sample size and follow-up time. It has been suggested that postoperative use of topical 
NSAIDs may results in prevention of PCO. ${ }^{14,24}$ At least in the adult cataract surgery, with two separate datasets, we did not observe this, even after accounting for potential confounders. Conversely, the use of topical NSAIDs seemed to result in higher Nd:YAG capsulotomy rates than with steroids.

There is also controversy on the use of NSAIDs vs steroids in the prevention of another cataract related complication, pseudophakic cystoid macular edema (PCME) ${ }^{25}$ A Cochrane review on the subject could not definitively comment on the equivalence or superiority of NSAIDs with or without steroids versus steroids alone for the prevention of PCME. ${ }^{26}$ The ESCRS PREMED study also did not show a difference in the incidence of clinically significant PCME between topical dexamethasone alone and bromfenac alone. ${ }^{27}$ According to a report by the American Academy of Ophthalmology (AAO), although NSAIDs might increase the speed of visual recovery, there is a lack of level I evidence that supports the long-term benefit of NSAID therapy to prevent vision loss from PCME. ${ }^{26}$ There are studies that suggest that in diabetic patients, topical steroids in combination with NSAIDs reduce the risk for PCME ${ }^{28}$ however these are not free of criticism. ${ }^{25}$

An extreme example of PCO development is found in the pediatric population that undergo cataract surgery where exaggerated capsular fibrosis is expected. In pediatric population studies, the inflammatory evidence for PCO formation is more robust. A recent study by Allam et al. demonstrated that when intraocular triamcinolone acetonide (TA) was used at the end of pediatric cataract surgery, a significantly lower rate of PCO was found compared to a group without TA. ${ }^{29} \mathrm{~A}$ similar observation was noted by Gupta et al. reporting significantly lower rates of PCO in 20 pediatric eyes with intraoperative TA injection. ${ }^{30}$ Although we did not include pediatric patients in our cohort, age was significantly associated with the development of PCO as younger patients required more Nd:YAG laser capsulotomies compared to older patients. Pediatric cataract cases could potentially have different inflammatory responses and mechanisms behind PCO formation compared to adult cataract surgeries. The conclusions of this study should therefore be interpreted with caution and cannot be directly generalized to pediatric cases.

In this study, outcomes of patients undergoing cataract extraction were assessed on a registry-based population of patients from the Helsinki University Hospital enabling a large-scale, real-world comparison between the different treatment regimens. The results show that treatment with steroids resulted in lower rates of clinically significant PCO compared with NSAIDs. The PCO preventive 
effect of steroids could be linked to several mechanisms. The first one is cyclooxygenase (COX) inhibition. ${ }^{13,14,19}$ In vitro, ex vivo and animal models showed that inhibition of COX reduced LEC proliferation associated with PCO formation. ${ }^{13,14}$ The COX enzyme produces several inflammatory mediators such as prostaglandins, which in turn induce inflammation and activate anti-apoptotic pathways, increasing LEC proliferation. ${ }^{13,14}$ Both NSAIDs directly and steroids indirectly, inhibit COX and this in turn decreases the inflammatory reaction and possibly limits LEC proliferation on the posterior capsule. ${ }^{31}$ While NSAIDs reduce mainly prostaglandins, steroids indirectly inhibit leukotriene production as well. Several in vitro, ex vivo and animal model studies showed that steroids and NSAIDs affect LEC production and migration differently; however the exact role of these anti-inflammatory drugs on PCO pathogenesis is not yet clear. ${ }^{20,21,32}$

Diabetes has been previously associated with increased severity of PCO, yet our results could not confirm this association. ${ }^{33}$ Another noteworthy finding was that pseudoexfoliation was not associated with higher PCO development risk, despite previous studies suggesting such an association. ${ }^{34}$ It is possible that due to the relatively low prevalence of pseudoexfoliation in this population, the study was underpowered to detect this association. The higher incidence of Nd:YAG capsulotomies among women could be explained by differences between genders toward medical care, as some studies suggest that women have higher utilization of health care services. ${ }^{35}$

The microstructure of Elschnig Pearl proliferation is different than fibrotic PCO. In Elschnig Pearls, cells show regular lenslike cellular structure, with globular structures protruding from the capsule. The microstructure of fibrotic PCO is characterized by multilayered aggregates of cells surrounded by extracellular matrix. ${ }^{36}$ Our study design does not enable distinguishing between the two. As clinically significant PCO was analysed based on the need for posterior capsulotomy, both fibrotic PCO and Elschnig Pearl proliferation were inherently included in the analysis.

This study has several limitations. First, given its retrospective design not all possible confounders could be accounted for or measured. Among them, surgical variation in capsule and subcapsular LEC cleaning, size of anterior capsulotomy, and degree of optic overlap by the capsulotomy, particularly given the relatively large number of surgeons $(\mathrm{N}=45)$ involved. Second, the incidence and degree of PCO was not directly measured but was analyzed based on the need for Nd:YAG capsulotomy following surgery, as an estimate of clinically significant PCO. Third, other surgical outcomes such as visual acuity postoperatively and prior to capsulotomy were not assessed. Fourth, these results should be generalized with caution to populations with dissimilar intraoperative, pre- 
or postoperative topical treatment regimens. Lack of standardization for agent and length of treatment is another limitation. This was intentional, as both type and length were almost uniform with over $95 \%$ treated with the same steroid, NSAID and for similar periods (as detailed in the Methods section). In addition, using real-world treatment patterns could make the outcomes more similar to those that can be expected elsewhere. Finally, a control group of patients not treated with any anti-inflammatory agent was not available for obvious reasons and so the independent effect of each agent could not be assessed.

To conclude, among patients undergoing uncomplicated cataract surgery, postoperative treatment with steroids was associated with lower PCO rates compared to treatment with NSAIDs, even after adjusting for confounders. Combination therapy of steroids and NSAIDs had no added benefit over steroid treatment alone. 


\section{Acknowledgments}

Funding: The study was supported by grants from the Finnish Eye Foundation, Finnish Ophthalmological Society, the Nissi Foundation, the Paulo Foundation, the Waldemar von Frenckell Foundation, and the HUS Specific Catchment Area (ERVA) Clinical Research Grants.

Financial Disclosures: The authors have neither proprietary nor commercial interests in any medications or materials discussed.

Other Acknowledgements: We want to thank Pasi Pöllänen, MD, PhD, Research Director of the Kymenlaakso Central Hospital, for his valuable comments on the research plan. 


\section{References}

1. Usmani B, Iftikhar M, Latif A, et al. Epidemiology of primary ophthalmic procedures performed in the United States. Can J Ophthalmol 2019;May 07, 2019 [Epub ahead of print].

2. Brandsdorfer A, Patel SH, Chuck RS. The role of perioperative nonsteroidal anti-inflammatory drugs use in cataract surgery. Curr Opin Ophthalmol 2019:30:44-9.

3. Ando H, Ando N, Oshika T. Cumulative probability of neodymium: YAG laser posterior capsulotomy after phacoemulsification. J Cataract Refract Surg 2003;29:(11):2148-54.

4. Elgohary MA, Dowler JG. Incidence and risk factors of Nd:YAG capsulotomy after phacoemulsification in non-diabetic and diabetic patients. Clin Exp Ophthalmol 2006;34:(6):52634.

5. Baratz KH, Cook BE, Hodge DO. Probability of Nd:YAG laser capsulotomy after cataract surgery in Olmsted County, Minnesota. Am J Ophthalmol 2001;131:(2):161-6.

6. Apple DJ, Peng Q, Visessook N, et al. Eradication of posterior capsule opacification: documentation of a marked decrease in Nd:YAG laser posterior capsulotomy rates noted in an analysis of 5416 pseudophakic human eyes obtained postmortem. Ophthalmology 2001;108:(3):505-18.

7. Kugelberg M, Wejde G, Jayaram H, et al. Posterior capsule opacification after implantation of a hydrophilic or a hydrophobic acrylic intraocular lens. J Cataract Refract Surg 2006;32:(10):162731.

8. Kossack N, Schindler C, Weinhold I, et al. German claims data analysis to assess impact of different intraocular lenses on posterior capsule opacification and related healthcare costs. J Public Health 2017;26:(1):81-90.

9. Berdeaux G. Cost of cataract surgery after implantation of three intraocular lenses. Clin Ophthalmol 2009:277. 
10. Coombes A, Seward H. Posterior capsular opacification prevention: IOL design and material. Br J Ophthalmol 1999;83:(6):640-1.

11. Slutzky L, Kleinmann G. Further Enhancement of Intraocular Open-Capsule Devices for Prevention of Posterior Capsule Opacification. Transl Vis Sci Technol 2018;7:(1):21.

12. Zhao Y, Yang K, Li J, et al. Comparison of hydrophobic and hydrophilic intraocular lens in preventing posterior capsule opacification after cataract surgery. Medicine 2017;96:(44):e8301.

13. Cortina P, Gomez-Lechon MJ, Navea A, et al. Diclofenac sodium and cyclosporin A inhibit human lens epithelial cell proliferation in culture. Graefes Arch Clin Exp Ophthalmol $1997 ; 235:(3): 180-5$.

14. Chandler HL, Barden CA, Lu P, et al. Prevention of posterior capsular opacification through cyclooxygenase-2 inhibition. Molecular vision 2007;13:677-91.

15. Karakosta A, Vassilaki M, Plainis S, et al. Choice of Analytic Approach for Eye-Specific Outcomes: One Eye or Two? Am J Ophthalmol 2012;153:(3):571-9.e1.

16. Shihan MH, Novo SG, Duncan MK. Cataract surgeon viewpoints on the need for novel preventative anti-inflammatory and anti-posterior capsular opacification therapies. Curr Med Res Opin 2019:1-.

17. Findl O, Buehl W, Bauer P, et al. Interventions for preventing posterior capsule opacification. Cochrane Database Syst Rev 2010.

18. Awasthi N. Posterior Capsular Opacification. Arch Ophthalmol 2009;127:(4):555.

19. Nibourg LM, Gelens E, Kuijer R, et al. Prevention of posterior capsular opacification. Exp Eye Res 2015;136:100-15.

20. Jiang J, Shihan MH, Wang Y, et al. Lens Epithelial Cells Initiate an Inflammatory Response Following Cataract Surgery. Invest Ophthalmol Vis Sci 2018;59:(12):4986. 
21. Symonds JG, Lovicu FJ, Chamberlain CG. Differing effects of dexamethasone and diclofenac on posterior capsule opacification-like changes in a rat lens explant model. Exp Eye Res 2006;83:(4):771-82.

22. Mansfield KJ, Cerra A, Chamberlain CG. Effects of dexamethasone on posterior capsule opacification-like changes in a rat lens explant model. Molecular vision 2004;10:728-37.

23. Zaczek A, Laurell C-G, Zetterström C. Posterior capsule opacification after phacoemulsification in patients with postoperative steroidal and nonsteroidal treatment. J Cataract Refract Surg 2004;30:(2):316-20.

24. Inan UU, Bozkurt E, Ozturk F, et al. Effect of diclofenac on prevention of posterior capsule opacification in human eyes. Can J Ophthalmol 2006;41:(5):624-9.

25. Grzybowski A, Kanclerz P. Controversies on the use of nonsteroidal antiinflammatory drugs and steroids in pseudophakic cystoid macular edema prophylaxis. J Cataract Refract Surg 2019;45:(12):1848.

26. Kim SJ, Schoenberger SD, Thorne JE, et al. Topical Nonsteroidal Anti-inflammatory Drugs and Cataract Surgery. Ophthalmology 2015;122:(11):2159-68.

27. Wielders LHP, Schouten JSAG, Winkens B, et al. European multicenter trial of the prevention of cystoid macular edema after cataract surgery in nondiabetics: ESCRS PREMED study report 1 . $J$ Cataract Refract Surg 2018;44:(4):429-39.

28. Laursen SB, Erichsen JH, Holm LM, et al. Prevention of macular edema in patients with diabetes after cataract surgery. J Cataract Refract Surg 2019;45:(6):854-69.

29. Allam G, Ellakkany R, Ellayeh A, et al. Outcome of pediatric cataract surgery with intraocular injection of triamcinolone acetonide: Randomized controlled trial. European Journal of Ophthalmology 2018;28:(6):633-8. 


\section{Journal Pre-proof}

30. Gupta R, Ram J, Sukhija J, et al. Outcome of paediatric cataract surgery with primary posterior capsulotomy and anterior vitrectomy using intra-operative preservative-free triamcinolone acetonide. Acta Ophthalmologica 2014;92:(5):e358-e61.

31. Goppelt-Struebe M, Wolter D, Resch K. Glucocorticoids inhibit prostaglandin synthesis not only at the level of phospholipase A2 but also at the level of cyclo-oxygenase/PGE isomerase. $\mathrm{Br} J$ Pharmacol 1989;98:(4):1287-95.

32. Mukai K, Matsushima H, Gotoh N, et al. Efficacy of ophthalmic nonsteroidal antiinflammatory drugs in suppressing anterior capsule contraction and secondary posterior capsule opacification. $J$ Cataract Refract Surg 2009;35:(9):1614-8.

33. Ebihara Y, Kato S, Oshika T, et al. Posterior capsule opacification after cataract surgery in patients with diabetes mellitus. J Cataract Refract Surg 2006;32:(7):1184-7.

34. Kuchle M, Amberg A, Martus P, et al. Pseudoexfoliation syndrome and secondary cataract. $B r J$ Ophthalmol 1997;81:(10):862-6.

35. Thompson AE, Anisimowicz Y, Miedema B, et al. The influence of gender and other patient characteristics on health care-seeking behaviour: a QUALICOPC study. BMC Fam Pract 2016;17:(1).

36. Marcantonio JM, Vrensen GFJM. Cell biology of posterior capsular opacification. Eye 1999;13:(3):484-8. 


\section{Figure and Table Legends}

Figure 1. Flow chart of the inclusion process

Flow chart of the inclusion process for patients following cataract extraction treated with different anti-inflammatory medications.

Figure 2. Kaplan-Meier survival plots of posterior capsulotomy free survival for patients treated with steroids compared to NSAIDs postoperatively

Survival plots of patients treated with steroids alone compared to NSAIDs alone. Hazard ratio (HR) for Nd:YAG capsulotomy for those treated with steroids (vs. NSAIDs) was 0.76 with $95 \%$ confidence interval 0.62-0.93, $P=0.009$. PCO, posterior capsule opacification.

Figure 3. Kaplan-Meier survival plots of posterior capsulotomy free survival for patients treated with combination therapy of steroids and NSAIDs compared with steroids alone Survival plots of patients treated with combination therapy of steroids and NSAIDs compared to steroids alone. Notice the crossing survival curves. Hazard ratio (HR) for Nd:YAG capsulotomy for those treated with steroids + NSAIDs (vs. steroids) was 1.11 with $95 \%$ confidence interval 0.68 1.80, $P=0.674$. $\mathrm{PCO}$, posterior capsule opacification.

Figure 4. Cox regression survival plots of Nd:YAG capsulotomy free survival for patients treated with steroids compared to NSAIDs postoperatively

Cox regression analysis of patients treated with steroids alone compared to NSAIDs alone adjusted for age, gender, existence of pseudoexfoliation and risk stratification. Hazard ratio (HR) for Nd:YAG capsulotomy for treatment with steroids (vs. NSAIDs) was 0.70 with $95 \%$ confidence interval 0.52-0.88, $P=0.001$. $\mathrm{PCO}$, posterior capsule opacification. 
Table 1. Patient Characteristics

NSAIDs, non-steroidal anti-inflammatory drugs. SD, standard deviation. Nd:YAG, neodymiumdoped yttrium aluminum garnet. *Preoperative risk assessment according to the referral to surgery, graded as low, medium, or high. ** Defined by subjective evaluation. 
Supplemental Figure 1. Kaplan-Meier survival plots of posterior capsulotomy free survival according to gender

Survival plots of patients according to gender following uncomplicated cataract removal surgery. Hazard ratio (HR) for Nd:YAG capsulotomy for females was 1.26 with $95 \%$ confidence interval 1.04-1.54, $P=0.018$. PCO, posterior capsule opacification.

Supplemental Figure 2. Cox regression survival plots of Nd:YAG capsulotomy free survival for patients treated with steroids compared to NSAIDs postoperatively of the second dataset

Cox regression analysis of patients treated with steroids compared to NSAIDs adjusted for age, and gender. HR for Nd:YAG capsulotomy for treatment with steroids (vs. NSAIDs) was 0.53 with $95 \%$ CI of $0.40-0.70, P<0.001$. PCO, posterior capsule opacification.

Supplement Table 1. Baseline characteristics of the second dataset

Data are given as mean \pm SD or proportion (\%). Dexamethasone (steroid) t.i.d. for 3 weeks or Diclofenac (NSAID) t.i.d. for 3 weeks as a primary anti-inflammatory treatment after cataract surgery. T.i.d.; three times a day. 
Table 1. Patient characteristics

\begin{tabular}{|c|c|c|c|}
\hline Variable & Steroids & NSAIDs & $\begin{array}{l}\text { Steroids + } \\
\text { NSAIDs }\end{array}$ \\
\hline $\mathrm{N}$ & 3,860 & 8,316 & 1,192 \\
\hline Age (years) $\pm(\mathrm{SD})$ & $73.1(9.6)$ & $73.1(9.6)$ & $74.3(10.3)$ \\
\hline Gender (female), n (\%) & $2,455(63.6)$ & $5,092(61.2)$ & $700(58.7)$ \\
\hline Pseudoexfoliation, n (\%) & $358(9.3)$ & $649(7.8)$ & $340(28.5)$ \\
\hline High Preop Grade*, n (\%) & $244(8.8)$ & $467(6.7)$ & $231(22.8)$ \\
\hline Poor Mydriasis**, n (\%) & $481(12.5)$ & $1,088(13.1)$ & $289(24.2)$ \\
\hline Pupil expansion device, $\mathrm{n}(\%)$ & $285(7.4)$ & $451(5.4)$ & $219(18.4)$ \\
\hline
\end{tabular}




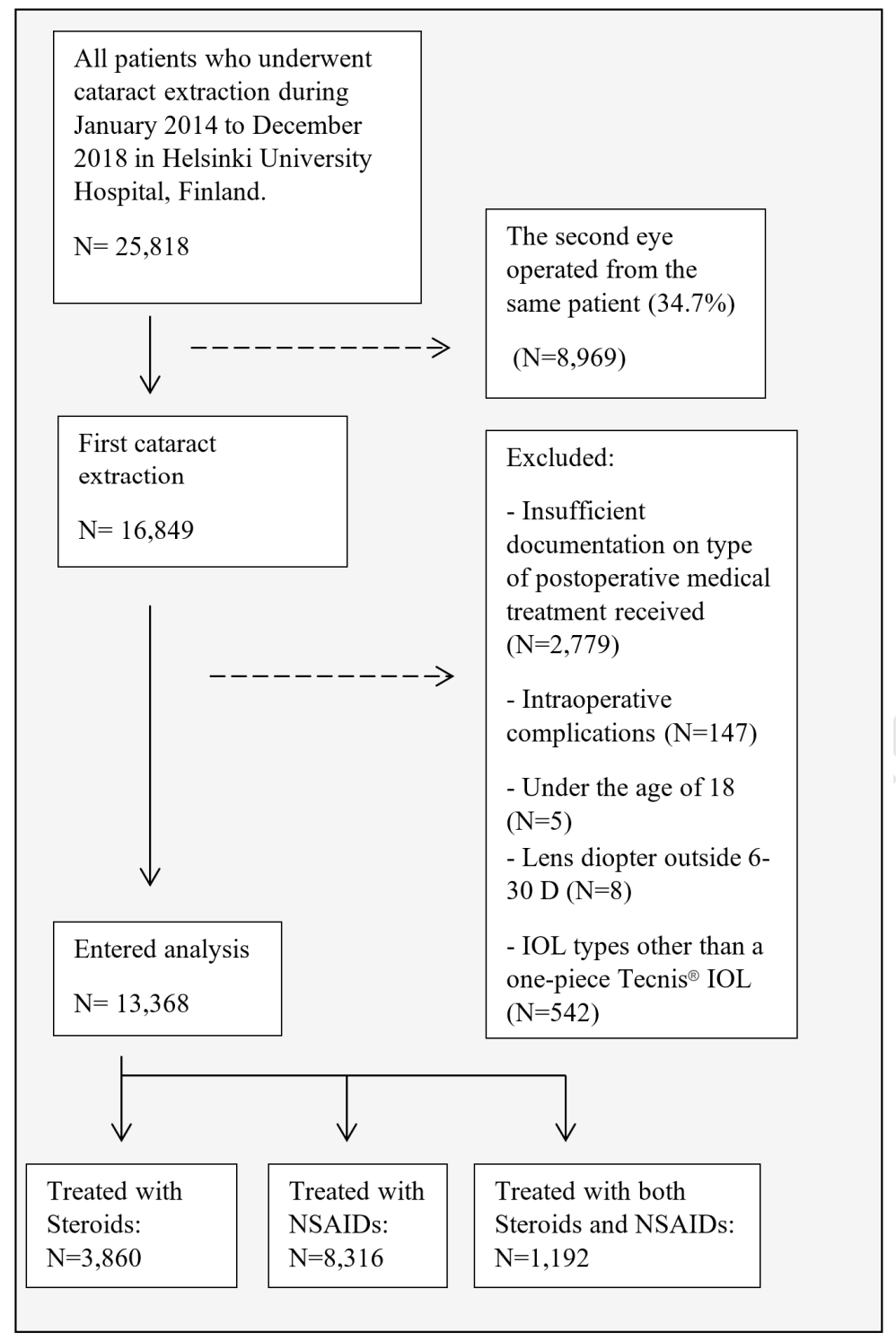


Number at Risk

$\begin{array}{lccccc}\text { Time-point } & \text { Baseline } & 12 \text { mo. } & 24 \text { mo. } & 36 \text { mo. } & 48 \text { mo. } \\ \text { Steroids } & 3860 & 2521 & 1950 & 1686 & 1013 \\ \text { NSAIDs } & 8316 & 5863 & 3247 & 1527 & 7\end{array}$




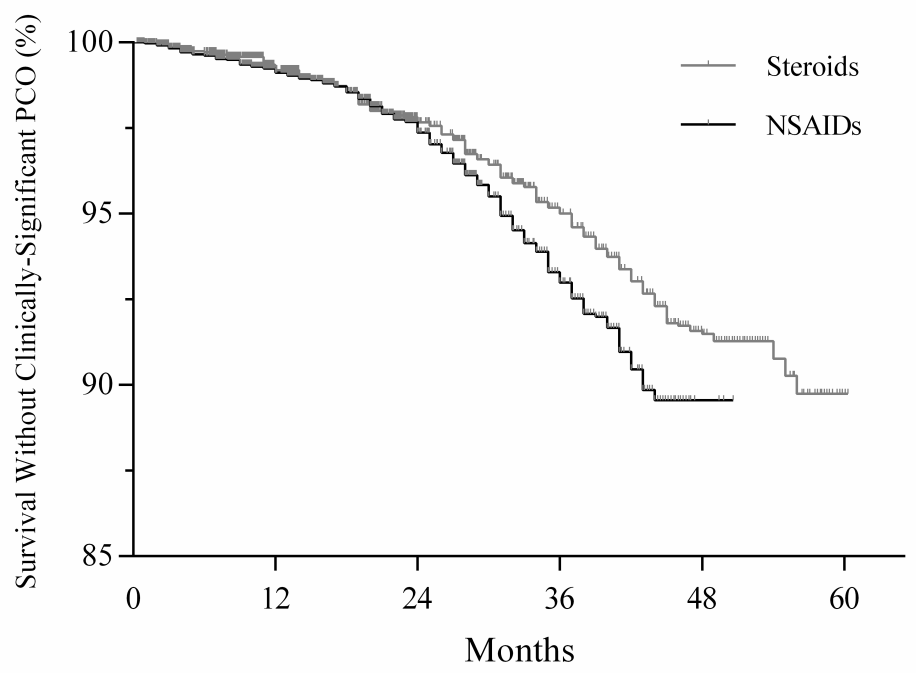


Number at Risk

\begin{tabular}{|c|c|c|c|c|c|c|}
\hline Time-point & & Baseline & $12 \mathrm{mo.}$ & 24 mo. & $36 \mathrm{mo.}$ & $48 \mathrm{mo}$ \\
\hline Steroids & & 3860 & 2521 & 1950 & 1686 & 1013 \\
\hline Steroids & + & 1192 & 628 & 226 & 64 & 0 \\
\hline
\end{tabular}

NSAIDs 


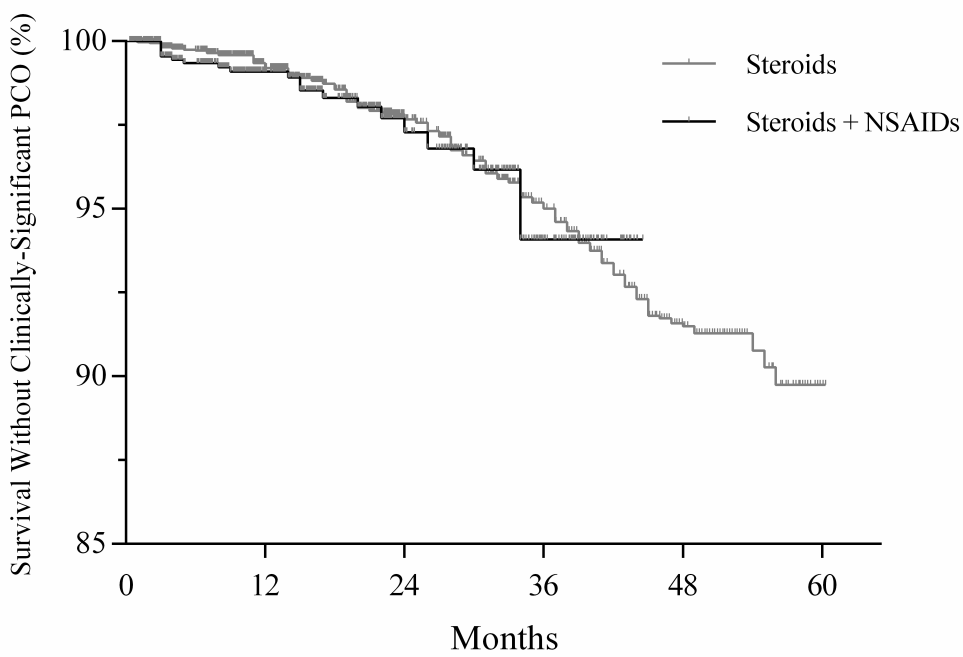




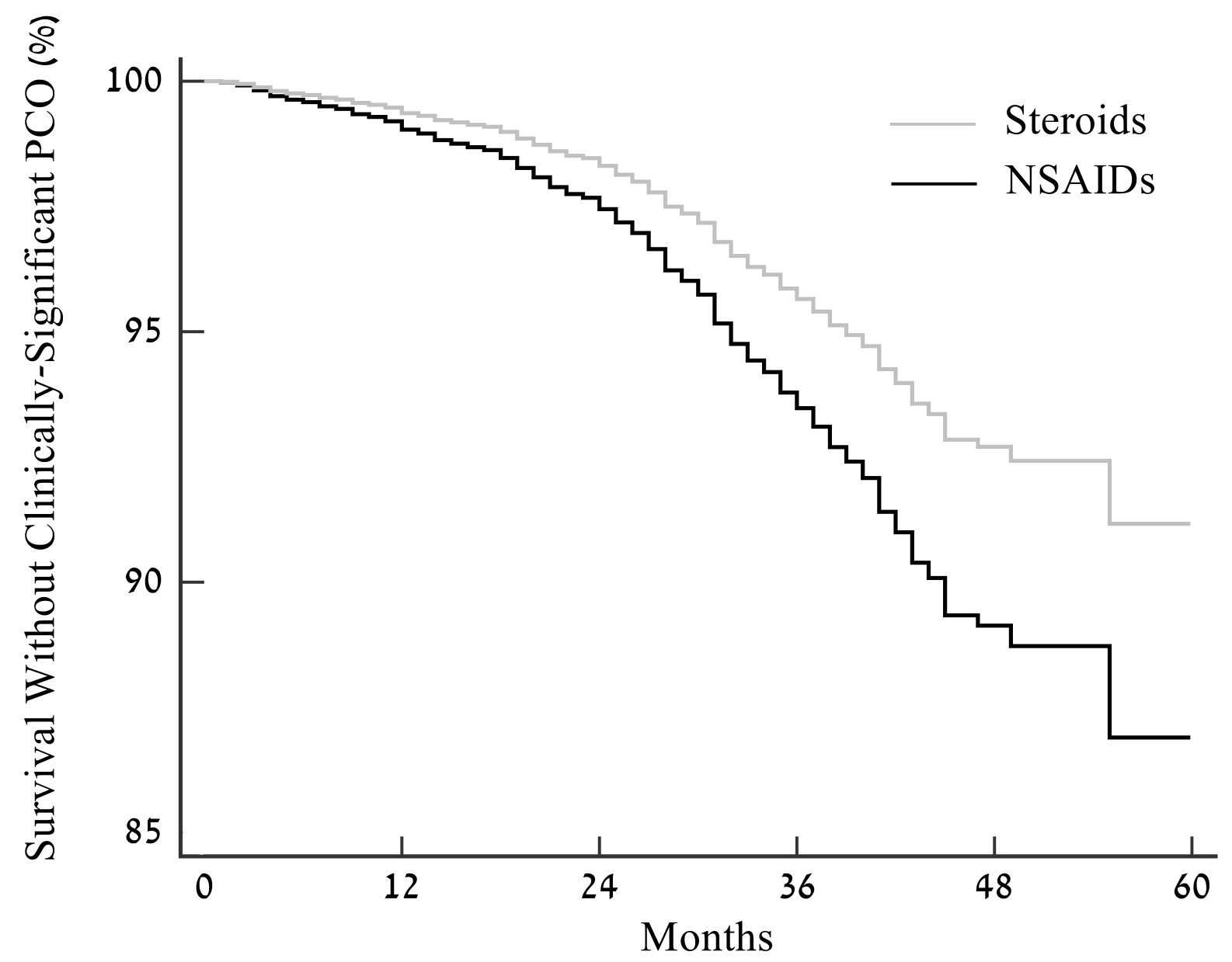




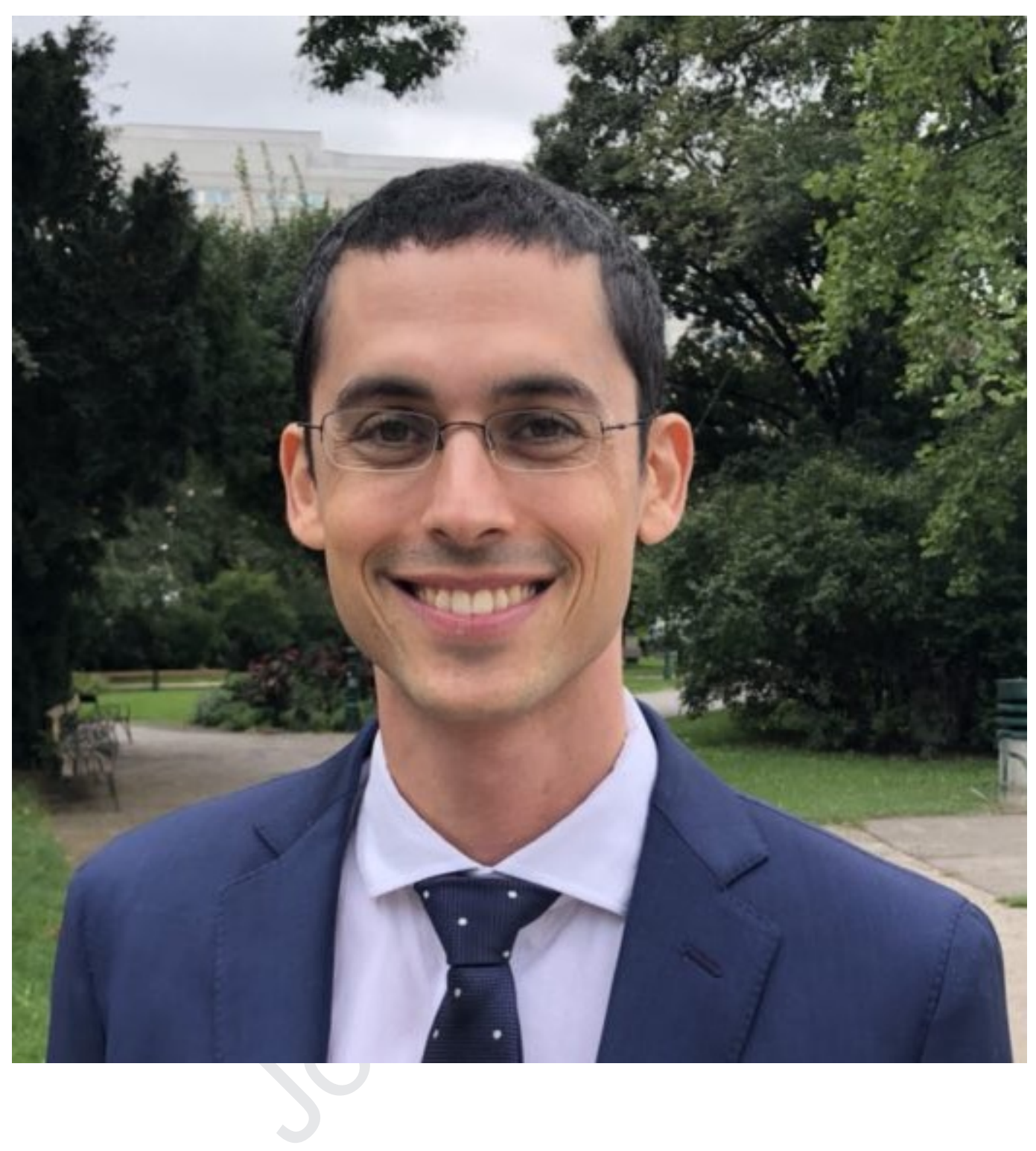




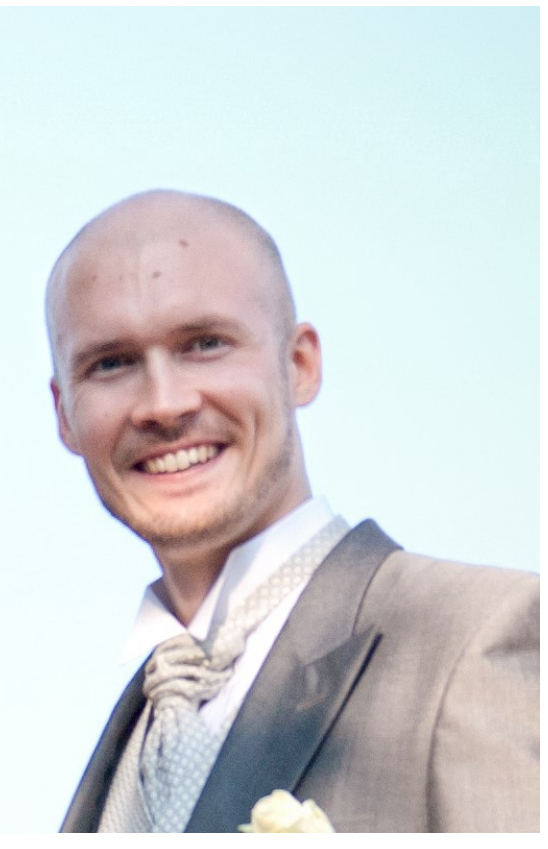




\section{Table of Contents Statement}

Previous piloting studies analysing the role of NSAIDs in the formation of PCO have shown contradictory results. It was suggested that postoperative use of topical NSAIDs may reduce formation of PCO. Nevertheless, no large-scale real-life evidence existed to date. In the adult cataract surgery, with two separate datasets, no observed protective role of topical antiinflammatory NSAIDs was seen over steroids. Conversely, the use of topical NSAIDs resulted in higher Nd:YAG capsulotomy rates than with steroids. 


\section{Credit Author Statement}

Raimo Tuuminen: Conceptualization, Methodology, Resources, Supervision, Writing - Review \& Editing. Ilkka Laine: Resources, Investigation, Writing - Review \& Editing. Uri Elbaz: Methodology, Investigation, Writing - Original Draft, Writing - Review \& Editing. Asaf Achiron: Supervision, Investigation, Formal analysis, Writing - Original Draft. Petteri Karesvuo: Resources, Investigation, Writing - Review \& Editing. Idan Hecht: Methodology, Formal analysis, Writing - Original Draft. 